\title{
Neutron-induced fission cross section of ${ }^{245} \mathrm{Cm}$ : New results from data taken at the time-of-flight facility n_TOF $_{-}$
}

M. Calviani, ${ }^{1,2, *}$ M. H. Meaze,${ }^{3, \dagger}$ N. Colonna, ${ }^{3}$ J. Praena, ${ }^{4}$ U. Abbondanno,${ }^{5}$ G. Aerts,${ }^{6}$ H. Alvarez,${ }^{7}$ F. Alvarez-Velarde,${ }^{8}$ S. Andriamonje, ${ }^{2,6}$ J. Andrzejewski, ${ }^{9}$ P. Assimakopoulos,${ }^{10,}$ L. Audouin, ${ }^{11}$ G. Badurek,${ }^{12}$ M. Barbagallo, ${ }^{3}$ P. Baumann ${ }^{13}$ F. Bečvár̆, ${ }^{14}$ F. Belloni, ${ }^{5,6}$ B. Berthier, ${ }^{11}$ E. Berthoumieux, ${ }^{6}$ F. Calviño, ${ }^{15}$ D. Cano-Ott, ${ }^{16}$ R. Capote, ${ }^{4,17}$ C. Carrapiço, ${ }^{6,18}$ P. Cennini, ${ }^{2}$ V. Chepel,,${ }^{19}$ E. Chiaveri, ${ }^{2}$ G. Cortes, ${ }^{15}$ A. Couture,${ }^{19}$ J. Cox,${ }^{19}$ M. Dahlfors, ${ }^{2}$ S. David,${ }^{11}$ I. Dillmann,${ }^{20}$ C. Domingo-Pardo, ${ }^{21}$ W. Dridi, ${ }^{6}$ I. Duran,${ }^{7}$ C. Eleftheriadis ${ }^{22}$ M. Embid-Segura,${ }^{8}$ L. Ferrant, ${ }^{11, \ddagger}$ A. Ferrari, ${ }^{2}$

R. Ferreira-Marques, ${ }^{23}$ K. Fujii, ${ }^{5}$ W. Furman, ${ }^{24}$ S. Galanopoulos, ${ }^{25}$ G. Giubrone, ${ }^{26}$ I. Gonçalves, ${ }^{18}$ E. Gonzalez-Romero, ${ }^{16}$ A. Goverdovski, ${ }^{27}$ F. Gramegna, ${ }^{1}$ C. Guerrero, ${ }^{16}$ F. Gunsing, ${ }^{6}$ B. Haas, ${ }^{28}$ R. Haight,${ }^{29}$ M. Heil, ${ }^{20}$ A. Herrera-Martinez, ${ }^{2}$ M. Igashira, ${ }^{30}$ E. Jericha, ${ }^{12}$ F. Käppeler, ${ }^{20}$ Y. Kadi, ${ }^{2}$ D. Karadimos, ${ }^{10}$ D. Karamanis, ${ }^{10}$ V. Ketlerov, ${ }^{27}$ M. Kerveno, ${ }^{13}$ P. Koehler, ${ }^{31}$ V. Konovalov, ${ }^{27}$ E. Kossionides, ${ }^{32}$ M. Krtička, ${ }^{14}$ C. Lampoudis, ${ }^{22}$ C. Lederer,${ }^{33}$ H. Leeb, ${ }^{12}$ A. Lindote, ${ }^{23}$ I. Lopes, ${ }^{23}$ M. Lozano, ${ }^{4}$ S. Lukic, ${ }^{13}$ J. Marganiec, ${ }^{9}$ S. Marrone,${ }^{3}$ T. Martínez, ${ }^{16}$ C. Massimi,${ }^{34}$ P. Mastinu, ${ }^{1}$ E. Mendoza, ${ }^{8}$ A. Mengoni, ${ }^{2,17}$ P. M. Milazzo, ${ }^{5}$ C. Moreau,${ }^{5}$ M. Mosconi,${ }^{20}$ F. Neves,${ }^{23}$ H. Oberhummer, ${ }^{12}$ S. O'Brien, ${ }^{19}$ J. Pancin, ${ }^{6}$ C. Papachristodoulou ${ }^{10}$ C. Papadopoulos,${ }^{25}$ C. Paradela, ${ }^{7}$ N. Patronis, ${ }^{10}$ A. Pavlik, ${ }^{33}$ P. Pavlopoulos,${ }^{35}$ L. Perrot, ${ }^{6}$ M. T. Pigni, ${ }^{12}$ R. Plag, ${ }^{20}$ A. Plompen, ${ }^{36}$ A. Plukis, ${ }^{6}$ A. Poch, ${ }^{15}$ C. Pretel, ${ }^{15}$ J. Quesada, ${ }^{4}$ T. Rauscher,${ }^{37}$ R. Reifarth, ${ }^{21}$ M. Rosetti, ${ }^{38}$ C. Rubbia, ${ }^{39}$ G. Rudolf,${ }^{13}$ P. Rullhusen, ${ }^{36}$ J. Salgado, ${ }^{18}$ C. Santos, ${ }^{18}$ L. Sarchiapone, ${ }^{1,2}$ R. Sarmento, ${ }^{18}$ I. Savvidis, ${ }^{22}$ P. Schillebeeckx, ${ }^{36}$ C. Stephan, ${ }^{11}$ G. Tagliente, ${ }^{3}$ J. L. Tain,${ }^{26}$ L. Tassan-Got,,${ }^{11}$ L. Tavora, ${ }^{18}$ R. Terlizzi,${ }^{3}$ G. Vannini, ${ }^{34}$ V. Variale, ${ }^{3}$ P. Vaz,${ }^{18}$ A. Ventura, ${ }^{38}$ D. Villamarin, ${ }^{16}$ M. C. Vincente, ${ }^{16}$ V. Vlachoudis, ${ }^{2}$ R. Vlastou, ${ }^{25}$ F. Voss,${ }^{20}$ S. Walter ${ }^{20}$ M. Wiescher, ${ }^{11}$ and K. Wisshak ${ }^{20}$

(n_TOF Collaboration ${ }^{\S}$ )

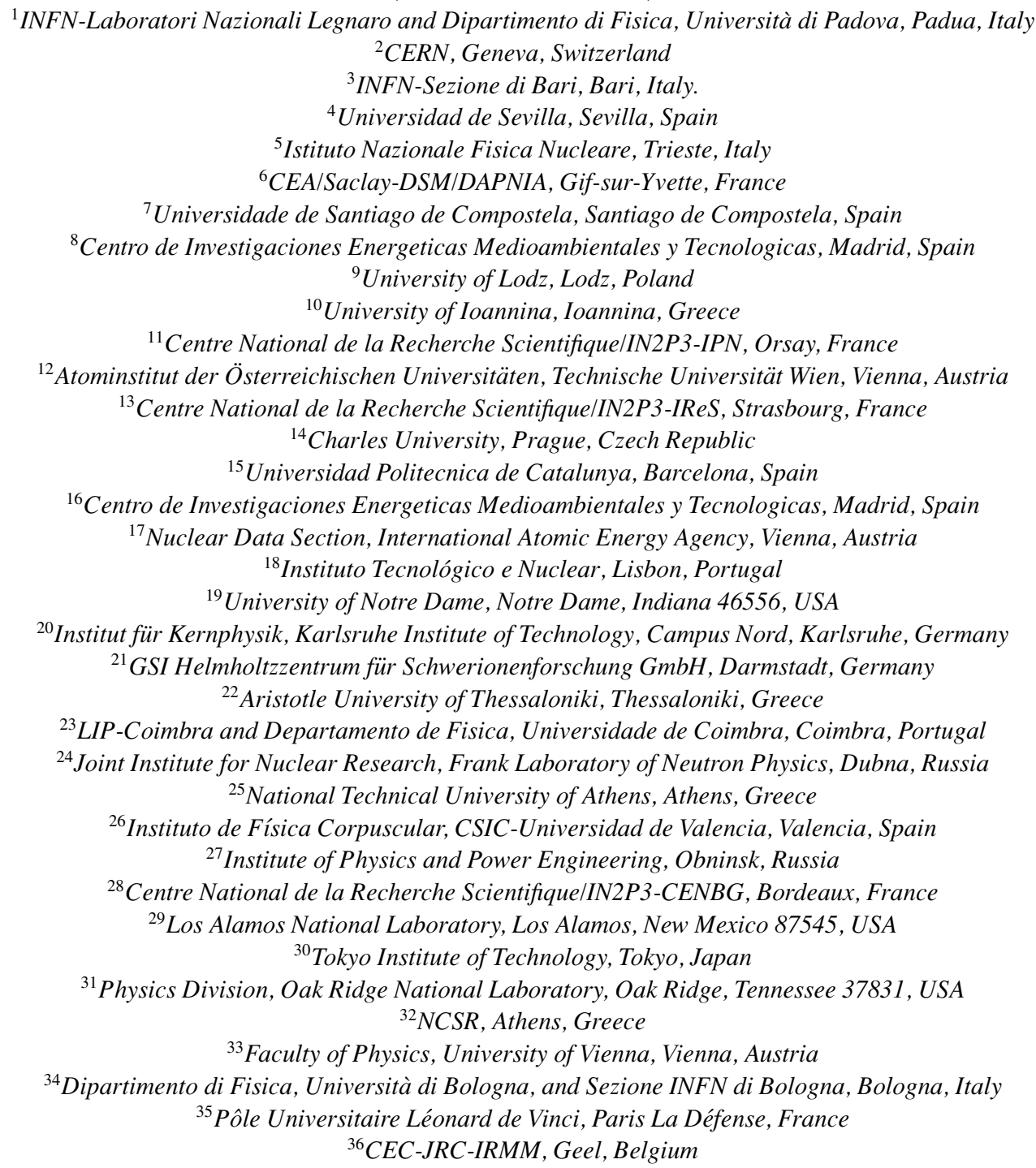




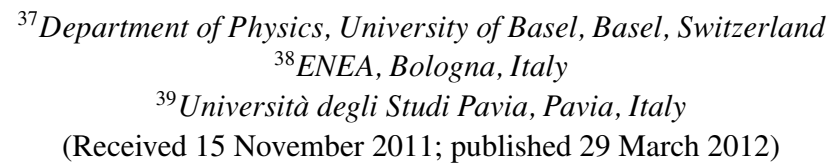

\begin{abstract}
The neutron-induced fission cross section of ${ }^{245} \mathrm{Cm}$ was measured at $\mathrm{n}_{-} \mathrm{TOF}$ in a wide energy range and with high resolution. The energy dependence, measured in a single measurement from $30 \mathrm{meV}$ to $1 \mathrm{MeV}$ neutron energy, has been determined with $5 \%$ accuracy relative to the ${ }^{235} \mathrm{U}(n, f)$ cross section. In order to reduce the uncertainty on the absolute value, the data have been normalized at thermal energy to recent measurements performed at ILL and BR1. In the energy range of overlap, the results are in fair agreement with some previous measurements and confirm, on average, the evaluated cross section in the ENDF/B-VII.0 database, although sizable differences are observed for some important resonances below $20 \mathrm{eV}$. A similar behavior is observed relative to JENDL/AC-2008, a reactor-oriented database for actinides. The new results contribute to the overall improvement of the databases needed for the design of advanced reactor systems and may lead to refinements of fission models for the actinides.
\end{abstract}

DOI: 10.1103/PhysRevC.85.034616

PACS number(s): 25.85.Ec, 28.65.+a, 27.90.+b

\section{INTRODUCTION}

Transuranium elements (TRU) such as $\mathrm{Np}, \mathrm{Pu}, \mathrm{Am}$, and $\mathrm{Cm}$ are built up as a result of multiple neutron captures and radioactive decays in all presently operating nuclear reactors based on the U/Pu nuclear fuel cycle. Some highly radioactive isotopes of these elements constitute the most important hazard for nuclear waste management. Several proposals have been made in recent years to reduce the radiotoxicity of nuclear waste containing TRU. Clearly, any kind of system designed to burn nuclear waste, critical or subcritical, thermal or fast, will need to be loaded with fuel containing a large fraction of TRU. The response of these systems (e.g., with respect to criticality) in the presence of TRU is directly linked to the fission cross sections of these isotopes. These fission cross sections are therefore key elements in assessing the strategy to be followed in detailed feasibility studies and in the engineering design of nuclear waste transmutation systems, i.e., Generation IV fast reactors or advanced nuclear waste burners [1].

As for most transuranium isotopes, the burnup of ${ }^{245} \mathrm{Cm}$ relies mostly on fission reactions. The competition with the neutron capture channel determines also the further buildup of the heavier curium isotopes and of heavier elements. In this context, accurate fission cross sections are required from thermal neutron energies to several MeV.

Besides these applications, the fission cross sections of the $\mathrm{Cm}$ isotopes are also of interest for improving the theoretical description of the fission process, particularly for the systematics of fission barriers. Fission cross sections are also needed as input for network calculations of $r$-process nucleosynthesis, where the reaction flow may be terminated by spontaneous, $\beta$-delayed, and neutron-induced fission of very neutron-rich actinides. Such a recycling may have a

\footnotetext{
*marco.calviani@cern.ch

†Present address: Department of Physics, University of Chittagong, Chittagong, Bangladesh.

${ }^{\ddagger}$ deceased

${ }^{\S}$ http://www.cern.ch/ntof
}

strong influence on the final $r$-process abundances [2,3]. The neutron-induced fission cross section of ${ }^{245} \mathrm{Cm}$ present large uncertainties of up to $50 \%$ in some energy regions, whereas sensitivity analyses for the most important advanced reactors indicate that accuracies better than $10 \%$ are needed [4].

Due to the relatively high $\alpha$ activity, which is related particularly to the unavoidable ${ }^{244} \mathrm{Cm}$ contamination of the sample material, only a few measurements of the ${ }^{245} \mathrm{Cm}(n, f)$ cross section have been performed so far. In 1969 Moore and Keyworth [5] reported data from $20 \mathrm{eV}$ to $3 \mathrm{MeV}$ by using a nuclear explosion as the neutron source. The measurement of Browne et al. [6] in 1978 at the $100 \mathrm{MeV}$ electron linear accelerator at Lawrence Livermore National Laboratory covered the neutron energy range between 0.01 and $35 \mathrm{eV}$. The short flight path of $3.6 \mathrm{~m}$ resulted in a poor energy resolution but permitted the use of a low sample mass of a few micrograms to reduce the $\alpha$-related background. Data taken by White et al. [7] soon afterward at the same facility extended the energy range up to $63.09 \mathrm{eV}$, with improved resolution. In this measurement, a ${ }^{245} \mathrm{Cm}$ sample with $99 \%$ enrichment was used. In the region of overlap, the two data sets differ significantly in various resonances. Recently, ${ }^{245} \mathrm{Cm}$ fission cross sections in the neutron energy range between $0.03 \mathrm{eV}$ and $20 \mathrm{keV}$ have been measured with a lead slowing-down neutron spectrometer at the Institute for Nuclear Research of the Russian Academy of Sciences [8]. Finally, the results of a new measurement performed at the GELINA neutron facility of Institute for Reference Materials and Measurements (IRMM), Geel, Belgium, have just become available [9]. Using a ${ }^{245} \mathrm{Cm}$ sample with $98.5 \%$ enrichment and a pair of $\mathrm{Si}-\mathrm{Au}$ surface barrier detectors, high-quality data from thermal to $100 \mathrm{eV}$ neutron energy were collected at the $9.3 \mathrm{~m}$ flight path, and a new resonance analysis was performed.

The limited amount of data and the discrepancies between them are reflected by the evaluations in the major cross-section libraries, ENDF/B-VII.0 [10], JEFF-3.1 [11], and JENDL-3.3 [12]. The cross sections in these libraries are all essentially based on the same evaluation [13], which relied on the data of Browne et al. [6] up to $20 \mathrm{eV}$ and on the data 
of Moore and Keyworth [5] at higher energy. A different approach was used in JENDL-4 [14], as well as in the special purpose file JENDL/AC-2008 [15], a specialized version of JENDL containing only data on actinides needed for advanced reactor technology (since the two files are equivalent for actinides, in the following we will only refer to the latter). In JENDL/AC-2008, the evaluation of the fission cross section of ${ }^{245} \mathrm{Cm}$ below $20 \mathrm{eV}$ was modified to better reproduce the thermal cross section and the results from time-of-flight measurements including the data of White et al. [7], which were not considered in Ref. [13].

The discrepancies between the various experimental results and the evaluated cross sections are a major problem for the new applications, which require uncertainties below $10 \%$. Further experimental data are therefore necessary to improve the accuracy of the $(n, f)$ cross section of ${ }^{245} \mathrm{Cm}$. To this purpose, a measurement was performed at the neutron facility $n_{-}$TOF at CERN, taking advantage of the wide neutron energy range, of the high resolution, and of the high instantaneous flux of this spallation neutron source [16]. This last property makes the n_TOF facility particularly convenient for measurements of radioactive isotopes since it minimizes the background related to the natural radioactivity of the samples.

The experimental setup used for the measurement is described in Sec. II. The resulting cross sections, which were obtained simultaneously from near thermal energies $(30 \mathrm{meV})$ up to $1 \mathrm{MeV}$, are presented in Sec. III, compared to previous data and to major evaluated data libraries. In Sec. IV the $R$-matrix analysis of the resonances is discussed, and the results are compared with previous evaluations.

\section{EXPERIMENTAL SETUP AND DATA ANALYSIS}

The experimental setup is based on the fission ionization chamber described in Ref. [17]. The signals were recorded with a flash analog-to-digital converter (ADC) [18] and analyzed to extract the neutron time of flight as well as the energy deposited by the fission fragments in the detector. The four curium samples used in the experiment were provided by the Institute of Physics and Power Engineering, Obninsk, and by the Joint Institute of Nuclear Research, Dubna. Thin layers of $\mathrm{CmO}_{2}$ were prepared on $100-\mu \mathrm{m}$-thick $\mathrm{Al}$ backings by the painting technique [19]. The masses and $\alpha$ activities are listed in Table I. As for all other samples in the measurement, the diameter of the $\mathrm{Cm}$ samples was $8 \mathrm{~cm}$ to match the diameter of the neutron beam used in the fission program at $\mathrm{n}_{-} \mathrm{TOF}$. The isotopic composition was measured by $\alpha$ spectroscopy, showing a $6 \%$ contamination of ${ }^{244} \mathrm{Cm}$. Because of its short half-life of $18 \mathrm{yr}$, this isotope is responsible for the large sample activities between 60 and $100 \mathrm{MBq}$. It also contributes significantly to the fission background due to its more than 200 times higher rate for spontaneous fission. Figure 1 shows the amplitude distribution of the digitized signals. The $\alpha$-particle and fission background were determined in runs without the neutron beam. The two large structures at low amplitude are due to $\alpha$ particles from the natural radioactivity of the sample and to their pileup, while the contribution of the spontaneous fission of ${ }^{244} \mathrm{Cm}$ is visible in the no-beam runs at all amplitudes.
TABLE I. Characteristics of the $\mathrm{CmO}_{2}$ samples used in the measurements discussed in this work. The overall activity of the samples is mostly due to the ${ }^{244} \mathrm{Cm}$ contamination.

\begin{tabular}{lccc}
\hline \hline Sample & Mass (mg) & Uncertainty (\%) & Activity (MBq) \\
\hline 1 & 0.367 & 1.3 & 66.1 \\
2 & 0.538 & 1.2 & 96.9 \\
3 & 0.407 & 1.3 & 73.3 \\
4 & 0.399 & 1.3 & 71.9 \\
\hline \hline
\end{tabular}

Because of the large $\alpha$ background and $\alpha$ pileup a rather high threshold had to be chosen for the pulse-height distribution. The value that maximizes the fission-to-background ratio was found around channel 80. Simulations of the fission fragment energy deposition in the gas (for details, see Ref. [20]) indicate that the detection efficiency obtained with this threshold is approximately $50 \%$.

Apart from the loss of statistics, the large background and the high threshold prevent the determination of the detection efficiency and other experimental effects (such as the deadtime correction) with sufficient accuracy. For this reason, an absolute normalization could not be performed for the present data set. A different approach, described below, had to be followed in order to extract accurate fission cross sections.

As illustrated in Fig. 1, even the high value of the threshold is not sufficient to fully reject the $\alpha$-particle background. Furthermore, the background from spontaneous fission has to be accurately determined. To minimize the statistical uncertainties, this time-independent residual background, which was measured without the neutron beam, was transformed into lethargy scale and fitted with the exponential relation $e^{a+b E}$, with the parameter $b$ being fixed to the value of -1.15129 expected from the time-to-lethargy conversion. With

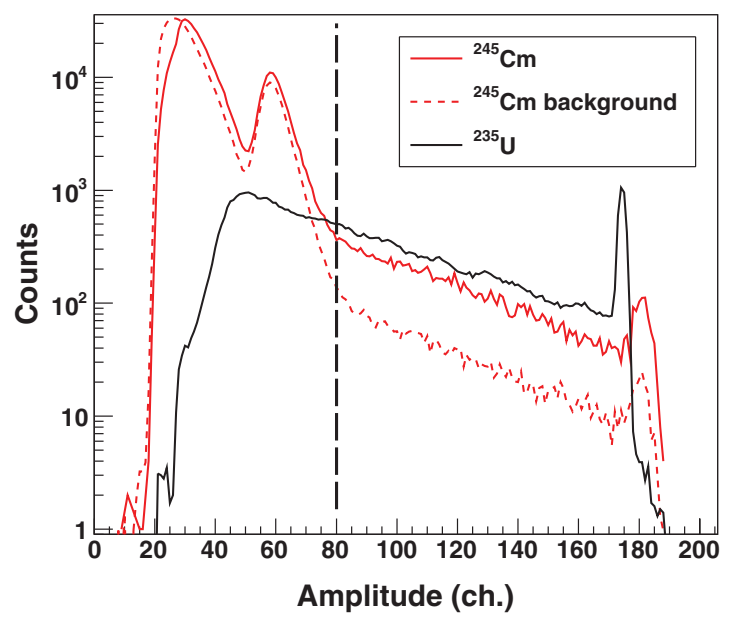

FIG. 1. (Color online) Amplitude distribution of signals recorded in the fission ionization chamber with the neutron beam [red (light grey) solid line] and during background runs with no beam [red (light grey) dashed line]. The structures at low amplitudes are produced by $\alpha$ particles and by $\alpha$-particle pileup. These backgrounds are missing in the amplitude distribution of ${ }^{235} \mathrm{U}$ (shown as a black solid line for comparison). The vertical dashed line at channel 80 represents the threshold used in the analysis. 
the parameter $a$ determined by the fit, the above expression was then used for background subtraction. A correction for the dead time (related to the overlap of two consecutive signals as defined in Ref. [17]) was applied to account for possible distortions in the energy dependence of the measured cross section. However, given the small count rate above the chosen threshold, the corrections are less than $1 \%$ in the investigated energy range.

The contribution of the ${ }^{244} \mathrm{Cm}(n, f)$ reaction to the measured fission yield was not subtracted below $500 \mathrm{keV}$ neutron energy because it was estimated to be less than $1 \%$. No correction was applied for the self-shielding effect, which is less than $0.1 \%$ even for the biggest resonances. Considering all sources of systematic uncertainties, the absolute cross section is characterized by uncertainties of $\sim 5 \%$ in the investigated energy range.

\section{NORMALIZATION AND RESULTS}

The cross section of the ${ }^{245} \mathrm{Cm}(n, f)$ reaction was determined relative to the ${ }^{235} \mathrm{U}$ reference sample, which was mounted in the same fission chamber. The procedure used in the analysis of the reference sample is described in detail in Ref. [20].

As mentioned before, the high threshold applied on the pulse-height distribution for the ${ }^{245} \mathrm{Cm}$ samples leads to a detection efficiency which is substantially different from that of the ${ }^{235} \mathrm{U}$ reference sample. An attempt to determine the difference by means of Monte Carlo simulations was abandoned because the correction factor depends on the amplitudeto-energy calibration, which could not be accurately defined due to the strong influence of the $\alpha$-particle background. Accordingly, the efficiency correction became too large to meet the intended accuracy of less than $10 \%$. The alternative approach was to use the thermal cross section for normalization. A thorough examination of the recommended thermal cross sections showed that earlier experimental data differed by up to $30 \%$. The recommended value in ENDF/B-VII.0 of $2142 \mathrm{~b}$ is based on the measurement of Browne et al. [6], which agrees within uncertainties with the results of Refs. [21,22] but is larger than reported in other measurements [23-25]. The JENDL/AC-2008 database recommends a lower thermal cross section of $2054 \mathrm{~b}$, which is based on the combined experimental results of Refs. [6] and [24]. Although the recommended values differ only by $5 \%$, the large discrepancy between the experimental results suggests that they are still uncertain by more than $10 \%$.

Therefore, the present data have been normalized to the thermal cross section reported in two recent measurements by Letourneau et al. [26], who obtained a value of $1943 \pm$ $65 \mathrm{~b}$ in a measurement at the Institut Laue-Langevin (ILL) high-flux reactor, Grenoble, France, and by Popescu et al. [27], who obtained $1951 \pm 18^{\text {stat }} \pm 81^{\text {syst }} \mathrm{b}$ in a measurement at the BR1 reactor of the Studiecentrum voor Kernenergie Centre d'Etude de l'énergie Nucléaire (SCK.CEN), Mol, Belgium. In view of the excellent agreement between both results, the weighted average of $1946 \pm 51 \mathrm{~b}$ has been adopted for normalization. Since the $\mathrm{n}_{-}$TOF data start at $30 \mathrm{meV}$ and do not extend down to $25 \mathrm{meV}$, an extrapolation has been used

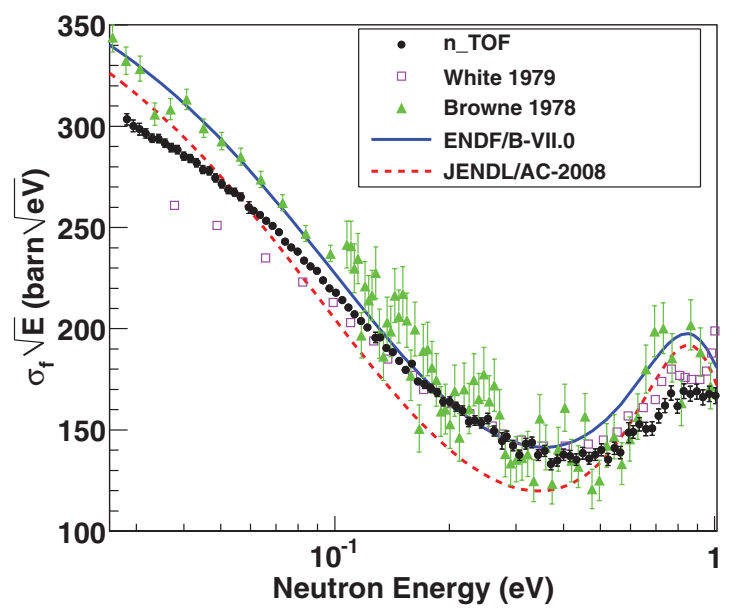

FIG. 2. (Color online) Energy-weighted neutron-induced fission cross section of ${ }^{245} \mathrm{Cm}$ from thermal energy to $1 \mathrm{eV}$. The $\mathrm{n}_{-} \mathrm{TOF}$ results (black dots), normalized to recent thermal cross-section measurements, are compared with previous data and with two evaluated data sets.

below $40 \mathrm{meV}$, similar to the one described in Ref. [20]. We note that the cross section determined in this way can easily be scaled if an improved value of the thermal cross section becomes available.

Figure 2 shows the energy-weighted cross section from thermal energy up to $1 \mathrm{eV}$. The results of the present work are compared with previous data and with the evaluated cross sections in ENDF/B-VII.0 and JENDL/AC-2008. In this region, the two previous measurements [6,7] show a different trend, with the biggest difference of $\sim 20 \%$ close to the thermal energy. The n_TOF data are intermediate between the two measurements below $100 \mathrm{meV}$ but are in reasonable agreement with data of White et al. [7] above that energy.

The comparison with evaluations shows some interesting features. The present results are not well reproduced either by ENDF/B-VII.0 or by JENDL/AC-2008 (which, by the way, does not reproduce any of the previous results either). In particular, the evaluated cross section in both databases shows a similar energy dependence but differs in the absolute cross section by approximately $20 \%$ up to $200 \mathrm{meV}$. Another point to note is that both evaluations overestimate the importance of the first resonance at $0.9 \mathrm{eV}$.

Figure 3 shows the $n_{-}$TOF results in the range between 1 and $10 \mathrm{eV}$, compared with previous data and evaluations. Previous results in this range show some discrepancies, with a higher cross section generally observed in the data of White et al. [7], particularly for the $7.5 \mathrm{eV}$ resonance. The $\mathrm{n}_{-} \mathrm{TOF}$ data are in reasonable agreement with this data set, except for the two biggest resonances. The shoulder on the right wing of the resonance at $2 \mathrm{eV}$, which does not seem evident in the data of Browne et al. [6], is instead confirmed by the present results.

Figure 4 shows the n_TOF results compared with previous data and evaluations between 10 and $17 \mathrm{eV}$. In this region the results of Browne et al. [6] suffer from the poor energy resolution of that measurement. For the $11.5 \mathrm{eV}$ resonance the $\mathrm{n}_{-} \mathrm{TOF}$ data fall between the two previous measurements, 


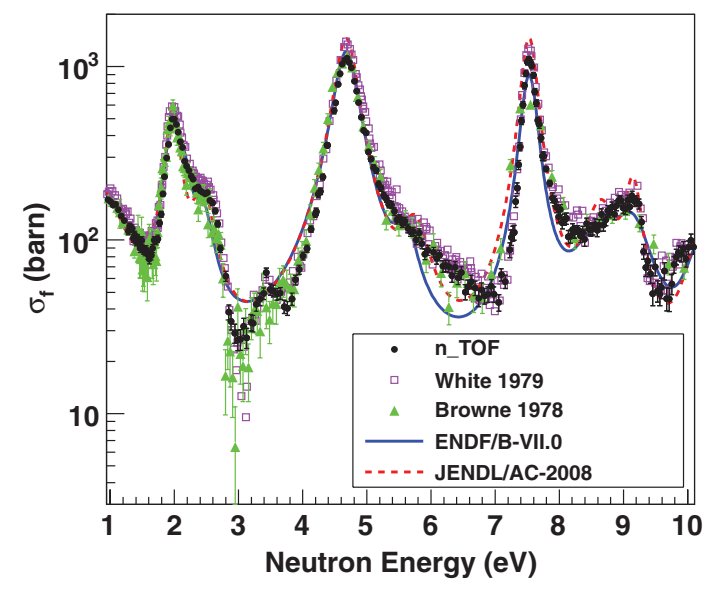

FIG. 3. (Color online) The present ${ }^{245} \mathrm{Cm}(n, f)$ cross sections of this work compared with previous data and evaluations in the energy range from 1 to $10 \mathrm{eV}$.

while the data of Ref. [6] are completely ruled out at higher energies by the $\mathrm{n}_{-} \mathrm{TOF}$ results. Apart from a systematic shift in the resonance energies, good agreement is observed again with the results of White et al. [7].

The large discrepancy between previous results is clearly reflected in the difference between the evaluations. In this region, ENDF/B-VII.0 strongly underestimates the resonance strengths, while JENDL/AC-2008 overestimates them slightly. Furthermore, the doublet in ENDF/B-VII.0 data around $15 \mathrm{eV}$ is clearly excluded by the present results. A preliminary resonance analysis shows that the $\mathrm{n}_{-} \mathrm{TOF}$ resonance strengths are $14 \%$ lower than those in Ref. [7], on average.

For neutron energies above $20 \mathrm{eV}$, data from Moore and Keyworth [5] are also available. In general, the $\mathrm{n}_{-} \mathrm{TOF}$ results are in very good agreement with this last data set, which was obtained with a collimated neutron beam from a nuclear explosion (Fig. 5). The agreement with the data of White et al. [7] is also reasonable. In this energy range the evaluated cross sections are essentially based on the results of Moore and Keyworth [5]. Consequently, they generally

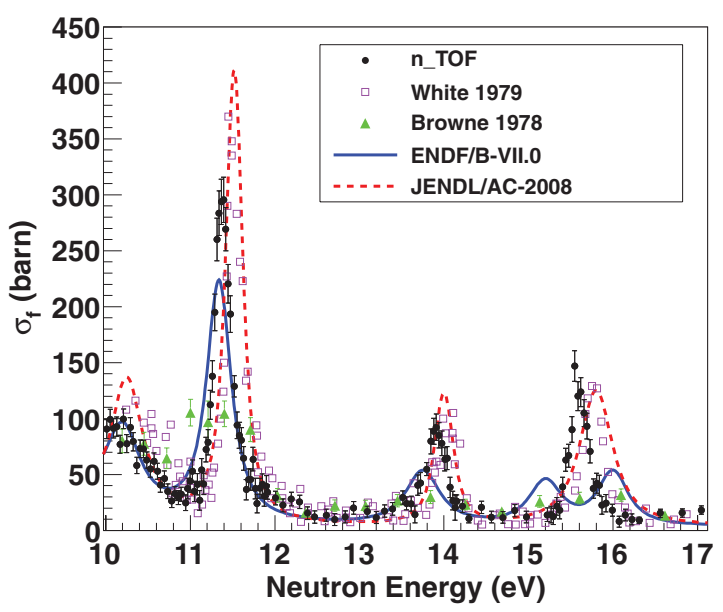

FIG. 4. (Color online) The ${ }^{245} \mathrm{Cm}(n, f)$ cross section of this work between 10 and $17 \mathrm{eV}$ neutron energy, compared with two previous data sets and with evaluations.

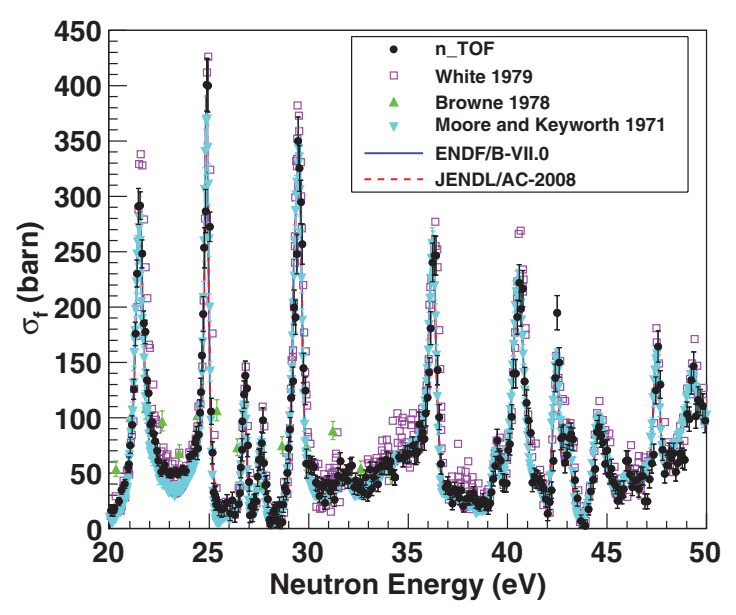

FIG. 5. (Color online) The ${ }^{245} \mathrm{Cm}(n, f)$ cross section of this work between 20 and $50 \mathrm{eV}$ neutron energy compared with previous measurements and with the ENDF/B-VII.0 and JENDL/AC-2008 evaluations. There is almost perfect agreement between the present results and the data of Ref. [5].

adequately reproduce the cross section measured at $\mathrm{n}_{-} \mathrm{TOF}$ as well.

The good agreement between the $\mathrm{n}_{-} \mathrm{TOF}$ results and the data in Ref. [5] is also confirmed at higher energy, as illustrated in Fig. 6. Because of the similar energy resolution the resonance structures are perfectly matched all the way up to $1 \mathrm{keV}$. The evaluations follow the resonance structures of the data below $100 \mathrm{eV}$, which can be considered as the current limit of the resolved resonance region (RRR) in the evaluated cross-section libraries. As discussed in Ref. [20] for the ${ }^{233} \mathrm{U}(n, f)$ reaction, the observed structures could be in some cases multiplets of resonances, particularly for neutron energies above a few hundred $\mathrm{eV}$.

An important confirmation of the reliability of the present results is provided by the comparison with the data recently obtained by Serot $e t$ al. at GELINA [9], shown in Fig. 7 from thermal to $100 \mathrm{eV}$ neutron energy. Apart from the difference

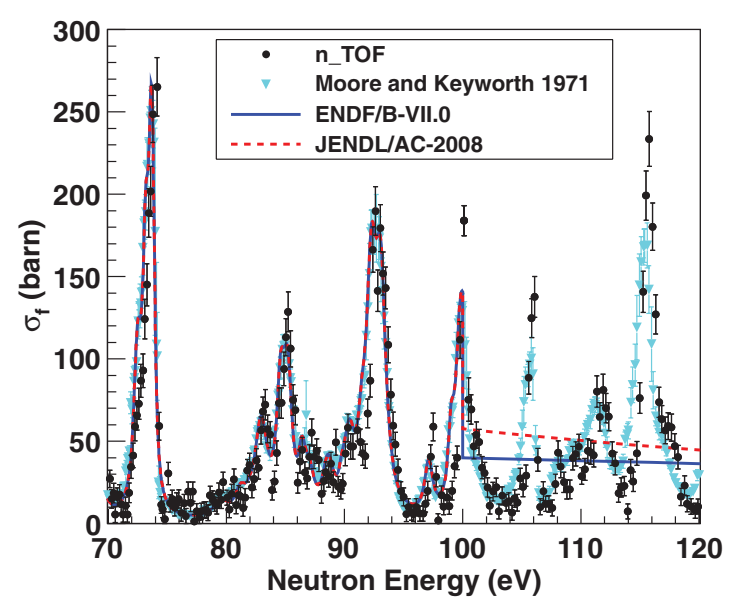

FIG. 6. (Color online) The ${ }^{245} \mathrm{Cm}(n, f)$ cross section in the neutron energy range from 70 to $120 \mathrm{eV}$. In this region, only one previous data set was available. 


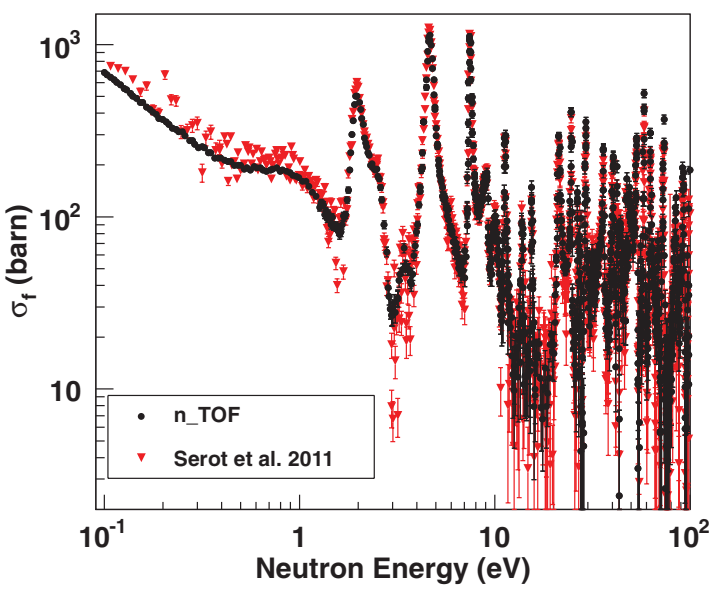

FIG. 7. (Color online) The present ${ }^{245} \mathrm{Cm}(n, f)$ cross section from thermal to $100 \mathrm{eV}$ neutron energy compared with recent results at GELINA [9].

below $1 \mathrm{eV}$, which is still within the systematic uncertainty of both data sets, and small discrepancies in the valleys of the first few resonances, there is a good agreement for all resonances, thus confirming the high level of confidence for the features described above.

The present high-resolution data in combination with the results of Serot et al. [9] and with the data of Moore and Keyworth [5] may permit the extraction of new and more accurate resonance (or pseudoresonance) parameters and the extension of the resonance region well above the current limit to roughly $300 \mathrm{eV}$ (see Sec. IV). Such an improvement in the knowledge of the resonant behavior of the cross section is important for accurate calculations of self-shielding effects in fuel elements, particularly in Generation IV fast reactors. At the same time, the data could provide more information on the average level density and other fundamental nuclear physics properties, leading to refinements of fission models for the actinides.

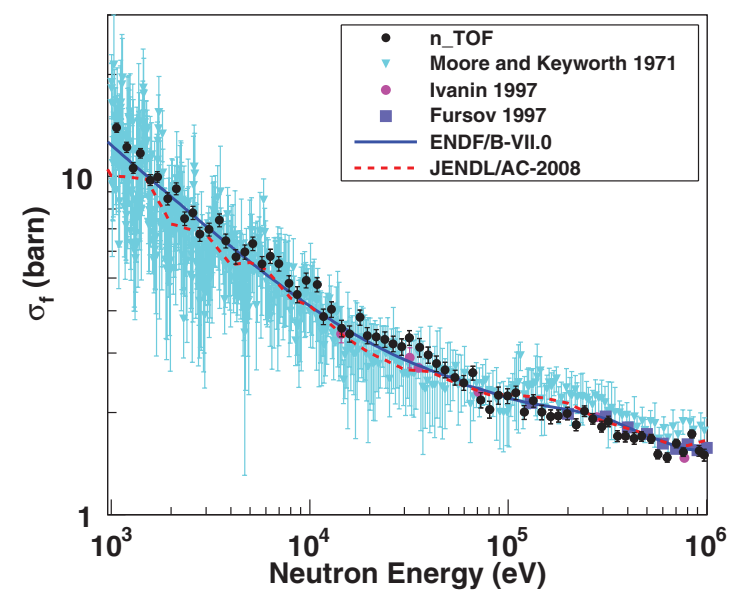

FIG. 8. (Color online) The ${ }^{245} \mathrm{Cm}(n, f)$ cross section in the unresolved resonance region from $1 \mathrm{keV}$ to $1 \mathrm{MeV}$ neutron energy, compared with the data of Refs. $[5,28,29]$ and with the evaluated data sets of ENDF/B-VII.0 and JENDL/AC-2008.
In the unresolved resonance region (URR), the $\mathrm{n}_{-} \mathrm{TOF}$ data are compared in Fig. 8 with those from Ref. [5] as well as with data from Fursov et al. [28] and Ivanin et al. [29]. Up to $100 \mathrm{keV}$ there is a fairly good agreement with all data sets. Around $100 \mathrm{keV}$, however, the data of Moore and Keyworth [5] show a kink, which from that point on gives rise to systematically higher cross sections. This behavior is not observed in the other two data sets, nor is it confirmed by the $\mathrm{n}_{-} \mathrm{TOF}$ data, suggesting that it could be due to an experimental artifact in the results of Ref. [5]. The cross section in the URR are listed in Table II with statistical uncertainties.

The comparison with evaluations shows relatively good agreement with ENDF/B-VII.0 and JENDL/AC-2008 in the unresolved energy range, with a slight preference for the former. Because the evaluated cross sections are based up to $\sim 200 \mathrm{keV}$ on few data sets, the $\mathrm{n}_{-}$TOF results are certainly useful for improving the evaluated data.

A more quantitative comparison between the $\mathrm{n}_{-} \mathrm{TOF}$ data, previous results, and evaluations can be performed by means of the resonance integral $R$ :

$$
R_{n, f}=\int_{E_{1}}^{E_{2}} \frac{\sigma_{n, f}(E)}{E} d E
$$

The calculated integrals for different energy regions are summarized in Table III. Because only the present data cover the entire energy range from thermal to $1 \mathrm{MeV}$, the comparison with previous results is limited to restricted energy regions. From thermal up to $500 \mathrm{meV}$, the integrated cross section of the present work falls in between the values obtained from previous results and evaluations. In the $0.5-20 \mathrm{eV}$ range, the cross sections measured at $\mathrm{n}_{-} \mathrm{TOF}$ are $5 \%$ and $13 \%$ lower than those reported in Refs. [6] and [7], respectively. The first difference is within the systematic uncertainty of the present data, while the second is clearly larger. The difference between the $\mathrm{n}_{-}$TOF results and those of Ref. [9] is 7\%, still within the quoted systematic uncertainties.

The best agreement is clearly between the integrated cross sections obtained at $\mathrm{n}_{-} \mathrm{TOF}$ and the data of Ref. [5] all the way from $20 \mathrm{eV}$ to $1 \mathrm{MeV}$. It should be noted, however, that at least for the $1 \mathrm{keV}$ to $1 \mathrm{MeV}$ neutron energy range, the good agreement on the integrated cross section masks a slightly different trend, with the data of Moore and Keyworth being lower at lower energies and slightly higher above $100 \mathrm{keV}$ (as shown in Fig. 8).

Compared to the resonance integrals calculated from the evaluated cross sections, the $\mathrm{n}_{-} \mathrm{TOF}$ values exhibit differences of $6 \%$ and $10 \%$ below $20 \mathrm{eV}$ with respect to ENDF/B-VII.0 and JENDL/AC-2008, respectively. Above $20 \mathrm{eV}$, there is excellent agreement between the present results and the evaluated cross section from both databases. While a maximum deviation of only $3 \%$ is observed with respect to ENDF/B-VII.0, the integrals obtained with the data of JENDL/AC-2008 differ by more than $5 \%$ from the $\mathrm{n}_{-} \mathrm{TOF}$ results above $1 \mathrm{keV}$. In summary, a revision of the evaluated nuclear data libraries for the ${ }^{245} \mathrm{Cm}(n, f)$ reaction is required, particularly below $1 \mathrm{eV}$ and for the first few resonances. 
TABLE II. The fission cross section of ${ }^{245} \mathrm{Cm}$ in the unresolved resonance region and related statistical errors.

\begin{tabular}{|c|c|c|}
\hline Neutron energy $(\mathrm{eV})$ & Cross section (b) & Statistical error (b) \\
\hline 1063.66 & 13.93 & 0.45 \\
\hline 1195.36 & 12.19 & 0.41 \\
\hline 1287.85 & 10.58 & 0.38 \\
\hline 1400.87 & 11.70 & 0.39 \\
\hline 1570.50 & 9.74 & 0.36 \\
\hline 1706.18 & 9.96 & 0.36 \\
\hline 1923.46 & 8.58 & 0.35 \\
\hline 2122.47 & 9.19 & 0.37 \\
\hline 2342.46 & 7.50 & 0.35 \\
\hline 2576.31 & 7.80 & 0.34 \\
\hline 2795.27 & 6.74 & 0.31 \\
\hline 3107.68 & 6.96 & 0.31 \\
\hline 3501.25 & 7.41 & 0.33 \\
\hline 3777.14 & 6.45 & 0.30 \\
\hline 4232.62 & 5.78 & 0.29 \\
\hline 4703.75 & 5.97 & 0.29 \\
\hline 5162.61 & 6.32 & 0.29 \\
\hline 5745.91 & 5.51 & 0.28 \\
\hline 6324.99 & 5.80 & 0.28 \\
\hline 6985.03 & 5.52 & 0.27 \\
\hline 7847.29 & 4.83 & 0.26 \\
\hline 8652.87 & 4.47 & 0.24 \\
\hline 9575.98 & 4.92 & 0.25 \\
\hline 10869.95 & 4.78 & 0.23 \\
\hline 11708.53 & 3.84 & 0.20 \\
\hline 12853.26 & 4.04 & 0.21 \\
\hline 14430.83 & 3.55 & 0.20 \\
\hline 15848.10 & 3.42 & 0.19 \\
\hline 17900.63 & 3.82 & 0.20 \\
\hline 19474.75 & 3.38 & 0.19 \\
\hline 21583.58 & 3.35 & 0.17 \\
\hline 23889.99 & 3.29 & 0.17 \\
\hline 26342.24 & 3.20 & 0.17 \\
\hline 29019.74 & 3.13 & 0.18 \\
\hline 31773.03 & 3.33 & 0.17 \\
\hline 35686.11 & 3.12 & 0.20 \\
\hline 39475.11 & 2.96 & 0.16 \\
\hline 43428.28 & 2.79 & 0.15 \\
\hline 48006.45 & 2.68 & 0.14 \\
\hline 53947.00 & 2.54 & 0.13 \\
\hline 59648.49 & 2.45 & 0.13 \\
\hline 66310.60 & 2.62 & 0.12 \\
\hline 72698.75 & 2.18 & 0.11 \\
\hline 80520.91 & 2.04 & 0.11 \\
\hline 89155.20 & 2.25 & 0.13 \\
\hline 99204.58 & 2.24 & 0.11 \\
\hline 109248.50 & 2.29 & 0.11 \\
\hline 120405.10 & 2.00 & 0.09 \\
\hline 133804.00 & 2.17 & 0.09 \\
\hline 147564.20 & 2.01 & 0.10 \\
\hline 163490.77 & 1.95 & 0.09 \\
\hline 178981.00 & 1.96 & 0.08 \\
\hline 198498.80 & 1.99 & 0.08 \\
\hline 219652.20 & 1.84 & 0.08 \\
\hline 242099.91 & 2.02 & 0.07 \\
\hline 266399.41 & 1.92 & 0.07 \\
\hline
\end{tabular}

TABLE II. (Continued.)

\begin{tabular}{lcc}
\hline \hline Neutron energy $(\mathrm{eV})$ & Cross section $(\mathrm{b})$ & Statistical error (b) \\
\hline 294989.00 & 1.82 & 0.07 \\
319148.50 & 1.88 & 0.07 \\
354762.69 & 1.70 & 0.06 \\
388847.09 & 1.70 & 0.06 \\
431067.00 & 1.68 & 0.07 \\
469301.91 & 1.70 & 0.06 \\
524493.50 & 1.67 & 0.05 \\
566595.00 & 1.51 & 0.05 \\
632016.81 & 1.47 & 0.05 \\
702688.63 & 1.62 & 0.05 \\
761827.88 & 1.53 & 0.04 \\
841674.31 & 1.73 & 0.05 \\
915319.13 & 1.54 & 0.06 \\
971360.81 & 1.50 & 0.06 \\
\hline \hline
\end{tabular}

\section{IV. $R$-MATRIX ANALYSIS}

In all evaluated data libraries the resolved resonance region extends up to $100 \mathrm{eV}$. In the ENDF/B-VII.0 evaluation, which is based on the work by Maslov et al. [13], the resonance parameters are extracted from the data by Browne et al. [6] from 0.01 to $20 \mathrm{eV}$ and by Moore and Keyworth [5] from 40 to $100 \mathrm{eV}$ and from the combination of both data sets in between. JEFF-3.1 and JENDL-3.3 use the ENDF/B-VII.0 evaluation and contain therefore identical resonance parameters. In JENDL/AC-2008, the parameters of levels below $20 \mathrm{eV}$ were instead evaluated on the basis of all three previous data sets (Refs. [5-7]) by using the multilevel Breit-Wigner formalism. Above $21 \mathrm{eV}$, resonance energies and partial widths are adopted from the evaluation of Maslov and are therefore identical to the values in ENDF/B-VII.0.

The present data indicate that ENDF/B-VII.0 and JENDL/AC-2008 need some revision, particularly in the thermal region and for the three major resonances below $20 \mathrm{eV}$. Furthermore, the high resolution of the $n_{-}$TOF beam allows one to extend the resonance analysis above the upper limit of the RRR in all current evaluations.

For these reasons an $R$-matrix analysis of the present data has been performed with the Bayesian code SAMMY [30] in the multilevel Breit-Wigner formalism. Corrections for the energy resolution of the neutron beam and for Doppler broadening due to the thermal motion of the target nuclei were taken into account in the fit. The Doppler broadening was modeled by a free gas at $300 \mathrm{~K}$. The effect of the potential scattering was considered by using a radius of $9.52 \mathrm{fm}$ [13]. The background was assumed to be zero, while the normalization was fixed to 1. Following the choice in Refs. [5], [6], and [13], all resonances were considered to be of $s$-wave type. We note that, as claimed in both evaluations, the average level spacing is too small to identify all individual resonances in the RRR and that the observed structures could be individual levels as well as aggregates of resonances (so-called pseudoresonances). This is particularly the case for neutron energies above a few tens of eV. In ENDF/B-VII.0, 88 positive and 3 negative 
TABLE III. The resonance integral (in barns) calculated for different energy regions from the $\mathrm{n}_{-}$TOF data, compared with the predictions of two major databases and with previous results. The quoted uncertainties refer only to the statistical errors. For White et al. [7] uncertainties are not available.

\begin{tabular}{|c|c|c|c|c|c|c|c|}
\hline Neutron energy & $\mathrm{n} \_\mathrm{TOF}$ & JENDL/AC-2008 & ENDF/B-VII.0 & Browne et al. [6] & White et al. [7] & $\begin{array}{c}\text { Moore and } \\
\text { Keyworth [5] }\end{array}$ & O. Serot et al. [9] \\
\hline $0.025-0.5 \mathrm{eV}$ & $2305.2 \pm 2.2$ & 2267 & 2447 & $2468.2 \pm 7.5$ & 2163.6 & & $2709.5 \pm 10.8$ \\
\hline $20-100 \mathrm{eV}$ & $109.2 \pm 0.4$ & 108 & 108 & & & $108.6 \pm 0.3$ & $103.3 \pm 0.5$ \\
\hline $0.1-1 \mathrm{keV}$ & $54.9 \pm 0.3$ & 52 & 56 & & & $54.3 \pm 0.2$ & $49 \pm 0.5$ \\
\hline $1 \mathrm{keV}$ to $1 \mathrm{MeV}$ & $28.3 \pm 0.2$ & 26.5 & 27.7 & & & $27.8 \pm 0.2$ & \\
\hline
\end{tabular}

energy resonances were assumed, while in JENDL/AC-2008, 86 positive resonances were complemented with only one resonance at negative energy.

The $\mathrm{n}_{-}$TOF data were fitted by leaving the resonance energy and $\Gamma_{\mathrm{f}}$ always free in the fit, while $\Gamma_{\gamma}$ was kept fixed due to its negligible impact. Because the fit of a fission resonance alone does not generally allow one to determine also the neutron width, $\Gamma_{\mathrm{n}}$ should be kept fixed, for each resonance, to the value determined in previous evaluations. However, in several cases, mostly below $20 \mathrm{eV}$, it was not possible to fit the resonance without changing $\Gamma_{\mathrm{n}}$.

The resonance parameters in JENDL/AC-2008 were adopted as initial values in the resonance fits below $100 \mathrm{eV}$. Above this energy, arbitrarily chosen initial values for $\Gamma_{\mathrm{f}}$ and $\Gamma_{\mathrm{n}}$ have been left free to vary in the fit. The same spin $(J=4)$ was assigned to all new resonances. Therefore, the resonance fits above $100 \mathrm{eV}$ can only provide accurate values for the fission kernels and not for the individual resonance parameters.

The SAMMY fits from thermal up to $100 \mathrm{eV}$ are presented in Figs. 9 and 10. The improvement relative to the most recent evaluations in ENDF/B-VII.0 and JENDL/AC-2008 is evident,

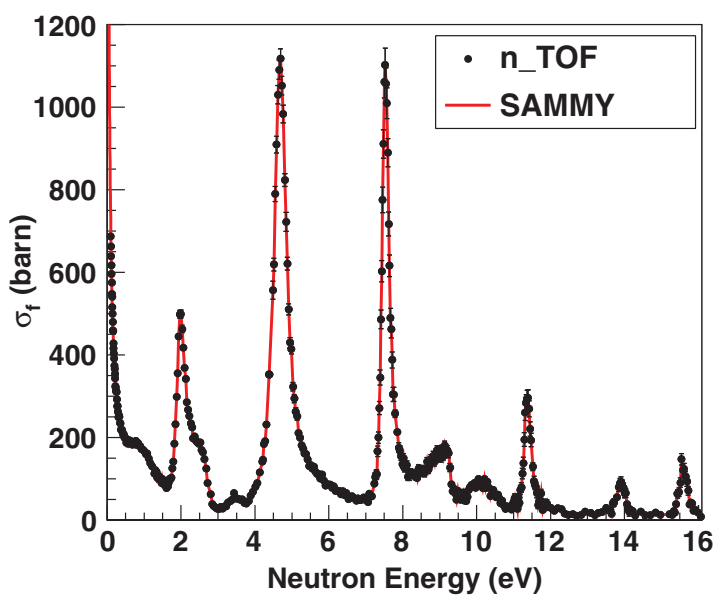

FIG. 9. (Color online) Fit of the n_TOF data up to $16 \mathrm{eV}$ neutron energy. The resonance parameters of JENDL/AC-2008 were assumed as initial values. The fit was performed leaving $E_{n}$ and $\Gamma_{\mathrm{f}}$ free to vary. For some resonances, $\Gamma_{\mathrm{n}}$ was also considered a free parameter, and a few resonances were added in this energy range to improve the fit. particularly for the strong resonances below $20 \mathrm{eV}$ (see, for comparison, Figs. 2-6). To reproduce the n_TOF data it was in some cases necessary to add new resonances and/or to change the resonance spins. For example, a new level at $3.5 \mathrm{eV}$ (with spin 4) was included to reproduce the tail of the $2 \mathrm{eV}$ resonance. In the present analysis, a total of 90 resonances (excluding the negative one) were counted below $100 \mathrm{eV}$, corresponding to an average level spacing of $1.11 \mathrm{eV}$.

The present $R$-matrix analysis demonstrates the need for a revision of the current resonance parameters, particularly below $20 \mathrm{eV}$. This is clearly demonstrated in Fig. 11 by the ratio of the fission kernels obtained from the present data and from the parameters given in the evaluated data libraries. While the kernels agree within $5 \%$ above $30 \mathrm{eV}$, thanks to the high accuracy of the nuclear explosion data of Moore and Keyworth [5], discrepancies of up to $50 \%$ are seen at lower energies, which need to be properly corrected.

The good energy resolution of the $n_{-}$TOF neutron beam allows one to observe resonances, or pseudoresonances, for neutron energies even above $100 \mathrm{eV}$, the limit of current evaluations. A preliminary analysis indicates that the range could be reasonably extended up to around $300 \mathrm{eV}$ with present statistics. Figure 12 shows a tentative fit performed in the

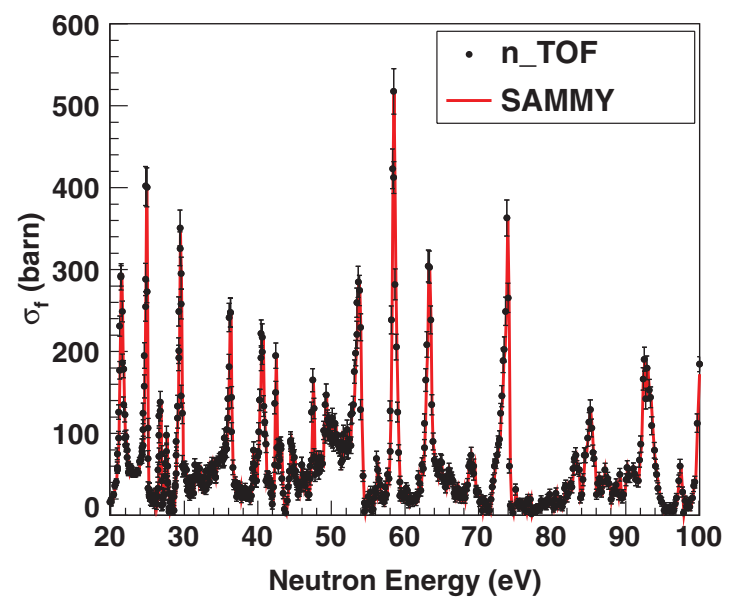

FIG. 10. (Color online) Fit of the $\mathrm{n}_{-}$TOF data from 20 to $100 \mathrm{eV}$ neutron energy. The strategy of the fit was the same as for the fits shown in Fig. 9. 


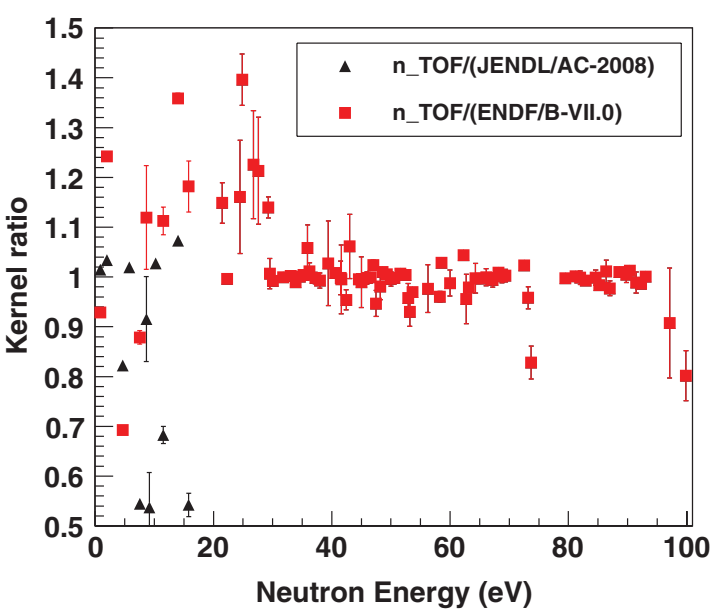

FIG. 11. (Color online) Ratio of the resonance kernels determined in this work and those determined from the resonance parameters of the two most recent evaluations. Above $20 \mathrm{eV}$ the resonance parameters in ENDF/B-VII.0 and JEND/AC-2008 coincide, and so does the ratio.

region between 100 and $200 \mathrm{eV}$ neutron energy. In this range, a total of 55 levels were found to be sufficient to reproduce the observed resonance structures.

\section{CONCLUSIONS}

The neutron-induced fission cross section of ${ }^{245} \mathrm{Cm}$ has been measured at $\mathrm{n}_{-} \mathrm{TOF}$ from $30 \mathrm{meV}$ to $1 \mathrm{MeV}$ neutron energy. Such a wide energy range is covered here in a single measurement. The energy dependence of the cross section could be determined with an uncertainty close to $3 \%$. However, it was not possible to obtain the absolute normalization with comparable accuracy because of the high threshold needed for reducing the large $\alpha$ background. Therefore, the data have been renormalized to the weighted average of the thermal cross section determined in two recent measurements. The combination of the uncertainties for background subtraction, normalization, and flux shape results in an overall systematic uncertainty around 5\% in the whole energy range. From thermal energies to $0.1 \mathrm{eV}$ the $\mathrm{n}_{-} \mathrm{TOF}$ data fall, on average, in between the only two previous time-of-flight measurements, while they clearly rule out one of these in the region above $10 \mathrm{eV}$. From $50 \mathrm{eV}$ up to $10 \mathrm{keV}$, the present results are in

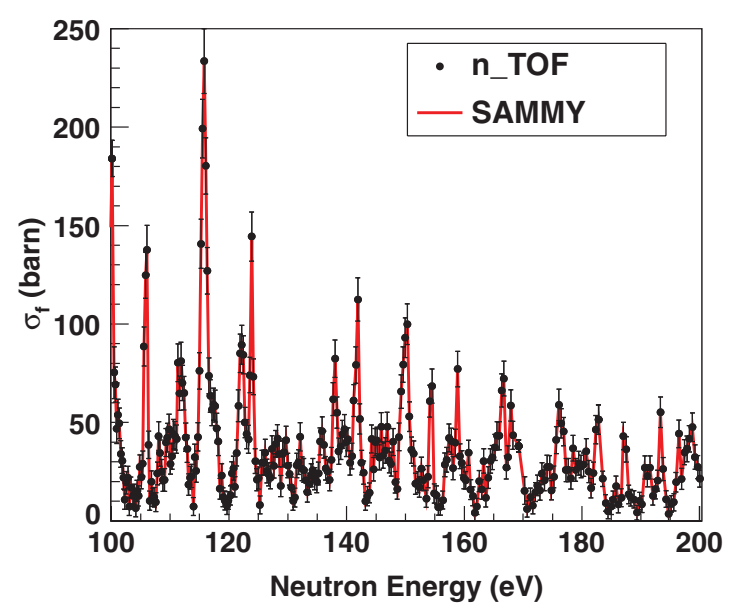

FIG. 12. (Color online) Fit of the n_TOF data from 100 to $200 \mathrm{eV}$ neutron energy. In this energy range no resonance analysis existed to date. The initial parameters for $E_{n}, \Gamma_{\mathrm{n}}, \Gamma_{\mathrm{f}}$, and the spin were arbitrarily chosen.

agreement with the only high-resolution data existing prior to this work [5], while at higher energies a slightly different energy dependence is observed relative to previous measurements.

Compared to the evaluated cross sections in the data libraries ENDF/B-VII.0 and JENDL/AC-2008, there is reasonably good agreement, i.e., within $\pm 5 \%$, for the resonance integrals. However, large differences exist for some resonances below $20 \mathrm{eV}$ as well as for the trend in the unresolved resonance region. A preliminary resonance analysis shows that the resonance parameters could be substantially improved and that the RRR could be extended to significantly higher energies. Accordingly, a revision of the evaluated fission cross section of ${ }^{245} \mathrm{Cm}$ is required, particularly for the resolved resonance region.

\section{ACKNOWLEDGMENTS}

This work was supported by the European Commission's 5th Framework Programme under Contract No. FIKW-CT2000-00107 (n_TOF-ND-ADS Project) and by the 7th Framework Programme under the ANDES project, Grant Agreement No. 249671.
[1] M. Salvatores, Nuclear Energy Agency, Report No. NEA/WPEC-26, 2008.

[2] I. V. Panov, E. Kolbe, B. Pfeiffer, T. Rauscher, K.-L. Kratz, and F.-K. Thielemann, Nucl. Phys. A 747, 633 (2005).

[3] J. Beun et al., Phys. Rev. C 77, 035804 (2008).

[4] G. Aliberti et al., Ann. Nucl. Energy 33, 700 (2006).

[5] M. S. Moore and G. A. Keyworth, Phys. Rev. C 3, 1656 (1971).

[6] J. C. Browne et al., Nucl. Sci. Eng. 65, 166 (1978).

[7] R. M. White et al., in Proceedings of the International Conference on Nuclear Cross Sections for Technology, edited by J. L. Fowler, C. H. Johnson, and C. D. Bowman (University of Tennessee, Knoxville, TN, USA, 1980), p. 496.
[8] A. A. Alekseev et al., At. Energy 106, 133 (2009).

[9] O. Serot, C. Wagemans, S. Vermote and J. Van Gils, J. Korean Phys. Soc. 59, 1896 (2011).

[10] M. B. Chadwick, P. Obložinský, M. Herman et al., Nucl. Data Sheets 107, 2931 (2006).

[11] The JEFF 3.1 Nuclear Data Library, edited by A. Koning, R. Forrest, M. Kellet, R. Mills, H. Henriksson, and Y. Rugama, JEFF Report No. 21, NEA Rep. No. 6190 (Nuclear Energy Agency, Paris, 2006).

[12] K. Shibata et al., J. Nucl. Sci. Technol. 39, 1125 (2002).

[13] V. M. Maslov, E. Sh. Sukhovitskij, Yu. V. Porodzinskij, A. B. Klepatskij, and G. B. Morovogskij, Evaluation of Neutron Data 
for Curium-245, INDC-BLR-003, International Atomic Energy Agency, Vienna, Austria (1996).

[14] K. Shibata et al., J. Nucl. Sci. Technol. 48, 1 (2011).

[15] O. Iwamoto et al., J. Nucl. Sci. Technol. 46, 510 (2009).

[16] U. Abbondanno et al., CERN, Report No. CERN/INTC-O-011, No. INTC-2002-037, 2002 (unpublished).

[17] M. Calviani et al., Nucl. Instrum. Methods Phys. Res., Sect. A 594, 220 (2008).

[18] U. Abbondanno et al., Nucl. Instrum. Methods Phys. Res., Sect. A 538, 692 (2005).

[19] L. V. Drapchinsky et al., Nucl. Instrum. Methods Phys. Res., Sect. A 438, 116 (1999).

[20] M. Calviani et al., Phys. Rev. C 80, 044604 (2009).

[21] H. Diamond et al., J. Inorg. Nucl. Chem. 30, 2553 (1968).

[22] K. D. Zhuravlev, N. I. Kroshkin, and A. P. Chetverikov, At. Energ. 39, 285 (1975).

[23] V. D. Gavrilov et al., At. Energ. 41, 185 (1976).

[24] R. W. Benjamin, K. W. Macmurdo, and J. D. Spencer, Nucl. Sci. Eng. 47, 203 (1972).
[25] J. Halperin, J. H. Oliver, and R. W. Stoughton, Oak Ridge National Laboratory, Report No. 4581, p. 73, 1970.

[26] A. Letourneau et al., in Proceedings of the 2nd EFNUDAT Workshop on Neutron Measurements, Theory and Applications, Budapest, Hungary, 2009, edited by T. Belgya, (Institute of Isotopes, Budapest, 2010), p. 81.

[27] L. Popescu et al., in 4th International Workshop on Nuclear Fission and Fission-Product Spectroscopy, edited by A. Chatillon, H. Faust, G. Fioni, D. Goutte, and H. Goutte, AIP Conf. Proc. No. 1175 (AIP, New York, 2009), p. 401.

[28] B. I. Fursov et al., in Proceedings of International Conference on Nuclear Data for Science and Technology, Trieste, Italy, 1997, edited by G. Reffo, A. Ventura, and C. Grandi (Italian Physical Society, Trieste, Italy, 1997), p. 488.

[29] A. Ivanin et al., in Proceedings of International Conference on Nuclear Data for Science and Technology, Trieste, Italy, 1997, edited by G. Reffo, A. Ventura, and C. Grandi (Italian Physical Society, Trieste, Italy, 1997), p. 664.

[30] N. M. Larson, Oak Ridge National Laboratory, Technical Report No. ORNL/TM-9179/7, 2006 (unpublished). 